\title{
Linx
}

LinX Revue des linguistes de l'université Paris X Nanterre

40 | 1999

Le statut d'unité lexicale

\section{A propos de noms de ponts}

\section{Leboutet}

\section{(Q) OpenEdition \\ Journals}

Édition électronique

URL : http://journals.openedition.org/linx/748

DOI : 10.4000/linx.748

ISSN : 2118-9692

\section{Éditeur}

Presses universitaires de Paris Nanterre

\section{Édition imprimée}

Date de publication : 1 juin 1999

Pagination : 71-77

ISSN : 0246-8743

\section{Référence électronique}

L. Leboutet, «A propos de noms de ponts », Linx [En ligne], 40 | 1999, mis en ligne le 22 juin 2012, consulté le 20 avril 2019. URL : http://journals.openedition.org/linx/748 ; DOI : 10.4000/linx.748

Ce document a été généré automatiquement le 20 avril 2019.

Département de Sciences du langage, Université Paris Ouest 


\section{A propos de noms de ponts}

\section{Leboutet}

\section{Les ponts sur la Seine à Paris}

1 A la suite de l'étude concernant les noms de rues, présentée dans ce numéro par B. Bosredon et I.Tamba, nous proposons une courte annexe relative à la désignation des ponts, en prenant pour exemple les ponts sur la Seine en région parisienne. « Un pont est une construction, un ouvrage reliant deux points séparés par une dépression ou par un obstacle ». (Petit Robert).

2 De tels ouvrages, de la passerelle à l'ouvrage d'art, en passant par les viaducs, les aqueducs, etc.. ont été construits à diverses fins. Dans cette note, nous ne considérerons que les appellations des types de ponts les plus utilisés, c'est-à-dire ceux qui relient des voies de communication urbaines destinées aux piétons, aux véhicules à deux et quatre roues, se rendant d'un endroit à un autre (la passerelle, pont étroit à garde-corps, étant réservée aux seuls piétons).

Comme il a été expliqué, dans l'article précédent, rues et routes ont reçu des noms, en France, comme en Europe. Il en est de même pour les ponts lorsque ceux-ci ne sont pas réservés au trafic ferroviaire, autoroutier ou métropolitain.

Prenons pour exemple la désignation des ponts sur la Seine à Paris et dans la proche banlieue Ouest. Nous nous déplacerons sur la Seine d'amont en aval, à partir du confluent de la Marne et de la Seine, (d'où le nom de «pont de Conflans » donné au pont reliant les deux villes d'Ivry-sur-Seine et de Charenton-le-Pont, situées à ce niveau).

Puis nous entrerons dans Paris en suivant le schéma joint à cet article. L'exercice paraîtra moins fastidieux que la lecture d'une énumération de 32 toponymes. En effet, il existe 32 ouvrages au-dessus de la Seine à Paris ayant reçu une dénomination, (les deux ponts routiers du périphérique n'en n'ayant pas été pourvus). Le schéma propose quelques éléments historiques propres à faire comprendre certaines appellations. Mais ce plan perspectif provient d'une « Sélection du Reader's Digest » qui date de 1971, (Paris et ses environs), de sorte que deux rectificatifs doivent être apportés : 
6 1/ Un nouveau pont a été construit ces toutes dernières années, le «pont Charles de Gaulle », qui relie le quai d'Austerlitz à la rue Van Gogh conduisant à la gare de Lyon.

7 2/ La passerelle dite « de Solférino » aboutissant au Jardin des Tuileries a disparu.

8 Ayant constaté que certains ponts des îles St Louis et de la Cité avaient conservé des noms anciens, nous allons inventorier cet ensemble à part. Son absence dans les énumérations qui vont suivre sera représentée par des points de suspension.

9 A partir de la série des toponymes rencontrés sur le schéma (exception faite des îles St Louis et de la Cité) nous relevons trois catégories syntaxiques de toponymes. Ce sont :

1/ pont+Adjectif ; 2/ pont+Nom propre ; 3/ pont+de+Nom propre.

11 On remarque que le mot adjectival est écrit avec une majuscule. On pourrait considérer ultérieurement qu'il fonctionne comme un nom propre. Voici le détail de ces nominations.

12 a/ Les ponts de Paris (sans ceux des îles St Louis et de la Cité).

\begin{tabular}{|l|l|l|}
\hline pont + Adjectif & pont + Nom propre & 3 \\
\hline National & Charles de Gaulle & pont + de + Nom propre \\
\hline Royal & Alexandre III & $\begin{array}{l}\text { de Tolbiac } \\
\text { de Bercy } \\
\text { d'Austerlitz }\end{array}$ \\
& Mirabeau & $\begin{array}{l}\text { des Arts } \\
\text { du Carrousel } \\
\text { de la Concorde } \\
\text { des Invalides } \\
\text { de l'Alma } \\
\text { d'Iéna } \\
\text { de Bir Hakeim } \\
\text { de Grenelle } \\
\text { du Garigliano }\end{array}$ \\
\hline Soit 2 & Soit 4 (avec la « passerelle Debilly ») & Soit 12 \\
\hline & &
\end{tabular}

b/ Les ponts des îles St Louis et de la Cité.

14 Aux désignations précédentes s'en ajoute une quatrième, héritée de l'histoire, et qui concerne les anciennes activités qui se déroulaient sur les ponts, (comme il en existe encore sur le pont de l'Arno à Florence, ou sur celui du Rialto, sur le Grand Canal, à Venise). Ce sont des désignations prenant la forme : "pont+au+Nom propre », telles que : «pont au Change», "pont au Double», dont le schéma accompagnateur rappelle l'origine, « change » et « double », étant ici devenus des noms propres.

La répartition des noms de ponts du Vieux Paris est donc la suivante : 


\begin{tabular}{|l|l|l|l|}
\hline 1 & 2 & 3 & 4 \\
pont + Adjectif & pont + Nom propre & pont + de + Nom propre & pont + au + Nom propre \\
\hline Petit (antéposé) & $\begin{array}{l}\text { Sully (2 ponts) } \\
\text { Neuf (postposé) }\end{array}$ & $\begin{array}{l}\text { de la Tournelle } \\
\text { de l'Archevêché } \\
\text { d'Arcole }\end{array}$ & $\begin{array}{l}\text { au Double } \\
\text { au Change }\end{array}$ \\
\hline $\begin{array}{l}\text { Notre-Dame } \\
\text { St Louis } \\
\text { St Michel }\end{array}$ & Soit 7 & Soit 3 & Soit 2 \\
\hline Soit 2 & \multicolumn{1}{|c|}{11} & & \\
\hline \hline Totalisons par colonne : & & & \\
\hline
\end{tabular}

Notons que l'on retrouve le type d'appellation ( $\left.\mathrm{N}^{\circ} 4\right)$, préservée au cours de l'histoire dans des noms de rues, par exemple à St Germain-en-Laye, ville natale de Henri II (1518) et de Louis XIV (1638), où existent encore la « rue au Pain », la « rue à la Farine ", et la « rue aux Prêtres ", ou, comme à Montmorency, berceau d'une illustre famille, où subsiste une « rue au Pain » et à Croissy-sur-Seine, une « rue au Mètre ».

En conclusion, l'ensemble des observations rassemblées dans les tableaux ci-dessus recoupe largement les conclusions de l'analyse exposée dans l'article précité puisque la double structure:"pont+Nom propre", et "pont+de+Nom propre» est, là aussi, majoritaire (26 exemples pour les 32 ponts).

c/ Outre la nature syntaxique de ces désignations de ponts, on peut chercher à quels événements ou à quels personnages elles réfèrent pour chacune des catégories $\mathrm{a} / \mathrm{et} \mathrm{b} /$.

Pour le premier groupe, elles renvoient au constructeur de l'ouvrage (il s'agit de la passerelle Debilly), ou à des personnages célèbres. Mais elles réfèrent surtout aux gloires des armées : Tolbiac, Austerlitz, Alma, Iéna, Bir-Hakeim, Garigliano. Plus simplement, les toponymes peuvent évoquer le site voisin ( Bercy), ou un monument proche, (L'Institut pour le pont des Arts, ou l'Hôtel des Invalides, pour le pont du même nom), ou un quartier (Grenelle).

En ce qui concerne la catégorie b/ des ponts du Vieux Paris, on trouve deux dignitaires, le constructeur du pont Marie, mais aussi des personnages religieux (dont Notre-Dame, la seule désignation féminine), personnages qui étaient absents de la catégorie a/ précédente. On relève des noms de lieu et de monument, et, finalement, les deux toponymes rappelant d'anciennes activités qui se déroulaient sur les ponts d'autrefois, comme dans les rues qu'ils prolongeaient.

\section{II- Les ponts dans les boucles de la Seine a la sortie de Paris}

On constate que pour désigner les ponts qui relient les différentes villes de la région, seules sont utilisées des données géographiques, ce qui paraît assez rationnel. Ces 
désignations figurent tant sur les panneaux routiers de signalisation qu'aux extrémités des ponts eux-mêmes. En partant de Paris et en se dirigeant vers l'Ouest proche, on observe que, à l'exception de la ville du Pecq, aucune commune de cette banlieue n'est traversée par la Seine. Il en résulte que le milieu du fleuve, et des îles qui peuvent s'y trouver), constitue généralement la limite entre deux villes riveraines opposées.

Pour la désignation du nom d'un pont, on choisit conventionnellement le sens centrifuge par rapport à Paris, dont le centre se trouvera situé à Notre-Dame. Le nom d'un pont sera celui de la deuxième ville rencontrée sur la Seine en s'éloignant de Paris. La convention s'applique aisément aux communes qui ne possèdent qu'un seul pont: Sèvres, SaintCloud, Suresnes, Villeneuve-la-Garenne, Bezons, Chatou, Croissy-sur-Seine, Bougival, Le Pecq, Maisons-Laffitte, où nous nous arrêterons.

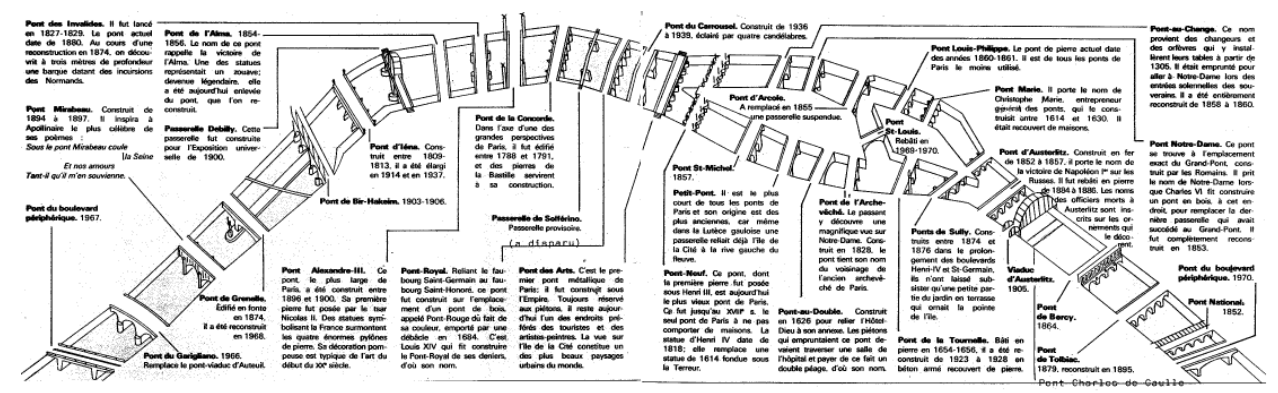

Il n'en est pas de même pour les villes possédant 2 ponts : Issy-les-Moulineaux, Puteaux, Argenteuil ; 3 ponts : Courbevoie, Asnières, Neuilly-sur-Seine, Gennevilliers, ou BoulogneBillancourt qui en possède 5 . Pour les appellations de ces divers ponts dans la ville, on utilise toutes les ressources fournies par les noms des communes limitrophes, ou par ceux des îles du voisinage, île de Billancourt, île de la Jatte à Neuilly, île de Croissy, etc.). Des toponymes de régions lointaines paraissent même parfois appelés en renfort, comme en témoigne le « pont de Lorraine » à Asnières. Mais ces désignations n'offrant plus d'intérêt syntaxique, nous arrêterons là cette investigation.

\section{BIBLIOGRAPHIE}

\section{Guides}

Guides bleus Hachette : Environs de Paris, 1971 ; Paris, Hauts de Seine, Guides bleus Hachette : Paris, Hauts de Seine, Seine St Denis, Val-de-Marne, 1968. Guide Baedeker Paris, 1992-1993; Paris, Baedeker, 1993.

Plans

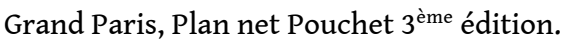

Paris par arrondissement Edition de la Police Nationale 1980.

Leconte banlieue, 240 communes de la Région Parisienne 1973. 
Cartes routières Michelin

1/Paris,12 ème édition ; 2/Banlieue de Paris

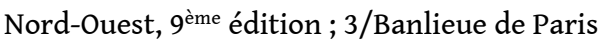

Sud- Ouest, $12^{\text {ème }}$ édition.

Ouvrages :

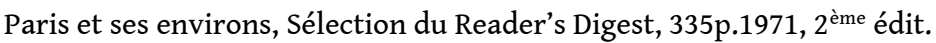

CATINAT J. C'est arrivé à Croissy-sur-Seine, 1971, Edit S.O.S.P.400 p.

MARIE A. St Cloud, 14 siècles d'histoire, 1979, Graphédis édit. 249 p.

PORTET M. St Cloud et les Hauts-de-Seine, 1966, Cortet édit. 244 p. 